\title{
Severe viral respiratory infections: are bugs bugging?
}

\author{
M Vissers ${ }^{1,2}$, R de Groot ${ }^{1,2}$ and G Ferwerda ${ }^{1,2}$
}

\begin{abstract}
Viral respiratory tract infections (RTI) pose a high burden on the youngest members of our society. Several risk factors are known for severe viral respiratory disease. However, a large proportion of the severe RTI cannot be explained by these risk factors. A growing body of evidence shows that the composition of the microbiota has a major influence on the training of both the mucosal and the systemic immune response and can thus potentially determine susceptibility for severe viral infections. In this review, we discuss the current evidence regarding the influence of bacterial colonization on the severity of viral respiratory infections.
\end{abstract}

\section{INTRODUCTION}

Acute respiratory tract infections (RTI) are the leading cause of mortality and morbidity in infants and young children. ${ }^{1,2}$ Both bacteria and viruses can cause serious RTI. Vaccination and the availability of antibiotics substantially reduced the mortality caused by bacterial RTI in developed countries. However, due to the limited availability of antiviral medications and effective vaccines the burden of viral RTI remains high. The leading cause of serious viral RTI in young children is respiratory syncytial virus (RSV), ${ }^{3}$ but influenza virus, rhinovirus, parainfluenza virus, adenovirus, and human metapneumovirus can also cause severe respiratory disease. $^{4,5}$

Children are frequently infected with these respiratory viruses, especially during the winter season. In most children, this leads to relatively mild symptoms, presenting as a common cold. However, some children have a more severe course of disease and develop lower respiratory tract symptoms, such as pneumonia and bronchiolitis. These children need to be hospitalized for supportive care and in severe cases mechanical ventilation is needed. This striking diversity in severity of infection is especially evident in RSV infections. Known risk factors to develop severe infection are prematurity, age ( $<6$ months), congenital heart disease and chronic lung disease (bronchopulmonary dysplasia), presence of siblings, and breastfeeding ( $<1$ month). ${ }^{3,6-8}$ The contribution of these risk factors to the development of severe disease is not fully understood. ${ }^{9}$ In addition, a large proportion of children with severe disease who required hospitalization are previously healthy and have no known risk factors. ${ }^{10,11}$

In recent years, a growing body of evidence has shown that colonization of mucosal tissues can influence the immune system both locally and systemically. We hypothesize that the composition of the microbiome may affect the severity of viral infection. This article aims to discuss the available evidence regarding the microbiome as a determinant for disease severity of viral RTI.

\section{THE MICROBIOME AFFECTS THE IMMUNE SYSTEM BOTH LOCALLY AND SYSTEMICALLY}

Mucosal surfaces of the human body provide residence to complex microbial ecosystems, together called the "microbiota". The presence of these bacteria is crucial for our well being. Microbiota have an important role in the digestive process in the intestines, produce vitamins, and provide a barrier to protect against translocation by and infection with pathogens. ${ }^{12}$

Vertebrates have co-evolved with bacteria present in their bodies for nearly half a billion years. Resident bacteria profoundly shape the immune system, while the immune system has to control the microbiota. This has resulted in a mutualistic and symbiotic partnership between the human immune system and these commensal microorganisms. ${ }^{13}$

${ }^{1}$ Department of Pediatrics, Laboratory of Pediatric Infectious Diseases, Radboud university medical center, Nijmegen, The Netherlands and ${ }^{2}$ Nijmegen Institute for Infection, Inflammation and Immunity, Radboud university medical center, Nijmegen, The Netherlands. Correspondence: G Ferwerda (Gerben.Ferwerda@radboudumc.nl) 


\section{Gut microbiota influence the immune system both locally and systemically}

The area in the human body with the highest diversity and density of microbes is the gastrointestinal tract. ${ }^{14}$ Although there is an enormous variety in taxa and composition between individuals, the gut microbiota is typically dominated by strict anaerobes like Firmicutes (e.g., Lactobacillus, Bacillus, and Clostridium) and Bacteroidetes (e.g., Bacteroides). ${ }^{15,16}$ In lower abundances Proteobacteria (e.g., Escherichia) and Actinobacteria (e.g., Bifidobacterium) can be found. ${ }^{15}$ The composition of the microbiota of the adult gut is mainly influenced by dietary patterns. ${ }^{17}$ However, broad-spectrum antibiotics, inflammation, or other stress inducers may influence the composition as well. ${ }^{17}$

The influence of the gut bacteria on the immune system has been extensively studied and reviewed. ${ }^{17-21}$ Disruption of the balance in the microbiota (dysbiosis) has been associated with inflammation-linked disorders, e.g., inflammatory bowel disease and airway allergies. A growing body of evidence suggests that the composition of gut commensals has systemic effects and influences the immune response at distant mucosal locations.

Clarke et al. ${ }^{22}$ have shown that peptidoglycan from the gut translocates to the bloodstream and to the bone marrow. This systemically present peptidoglycan primes the immune system, enhancing neutrophil killing of two important pathogens, Streptococcus pneumoniae and Staphylococcus aureus. Commensal gut bacteria influence the balance of T-cell subsets, which reaches far beyond the extent of the intestinal lamina propria. In germ-free (GF) mice, colonization with segmented filamentous bacteria skews the immune system towards a proinflammatory response, inducing T helper type 17 (Th17) cells and some Th1 cells leading to arthritis and experimental autoimmune encephalomyelitis. ${ }^{23,24}$ In contrast, colonization with certain Clostridial strains skews towards an antiinflammatory response, inducing regulatory $\mathrm{T}$ cells $\left(\mathrm{T}_{\text {regs }}\right)$, which reduce serum immunoglobulin $\mathrm{E}$ (IgE) responses after immunization. ${ }^{25}$ Colonization with polysaccharide A-producing Bacteroides fragilis results in higher numbers of circulating $\mathrm{CD} 4+\mathrm{T}$ cells and a higher Th1 response in circulation. $^{26}$

Multiple studies have shown that the gut microbiota also have an influence on the immune response of the airways. ${ }^{27}$ The hygiene hypothesis proposes that disruption of the gut microbiota by, e.g., antibiotics, dietary changes, or a reduction in infections due to decreased exposure, induces a disturbance of the immunological tolerance, resulting in enhanced allergic airway diseases. This is supported by experiments showing that antibiotics disturb the microbiota of the gut of mice, which subsequently induces a reduction in Th1 response and a more severe allergic response in the airways. ${ }^{28}$

Collectively, these data suggest that the commensals of the intestines are crucial for training of the immune system both locally and systemically (Figure 1).

\section{Microbiota of the respiratory tract}

Respiratory viruses enter the human body through the upper respiratory tract. The bacteria present in the airways may therefore be of importance for the host response towards a viral infection. At present, there are a limited number of papers studying respiratory tract microbiota in healthy adults. An overview of these papers can be found in Table 1 .

It has been shown that Firmicutes and Actinobacteria dominate the nostrils. These are mainly Staphylococcus, Corynebacterium, and Propionibacterium on genus level, which are typical skin lineages. ${ }^{32,33}$ The oropharynx contains, on

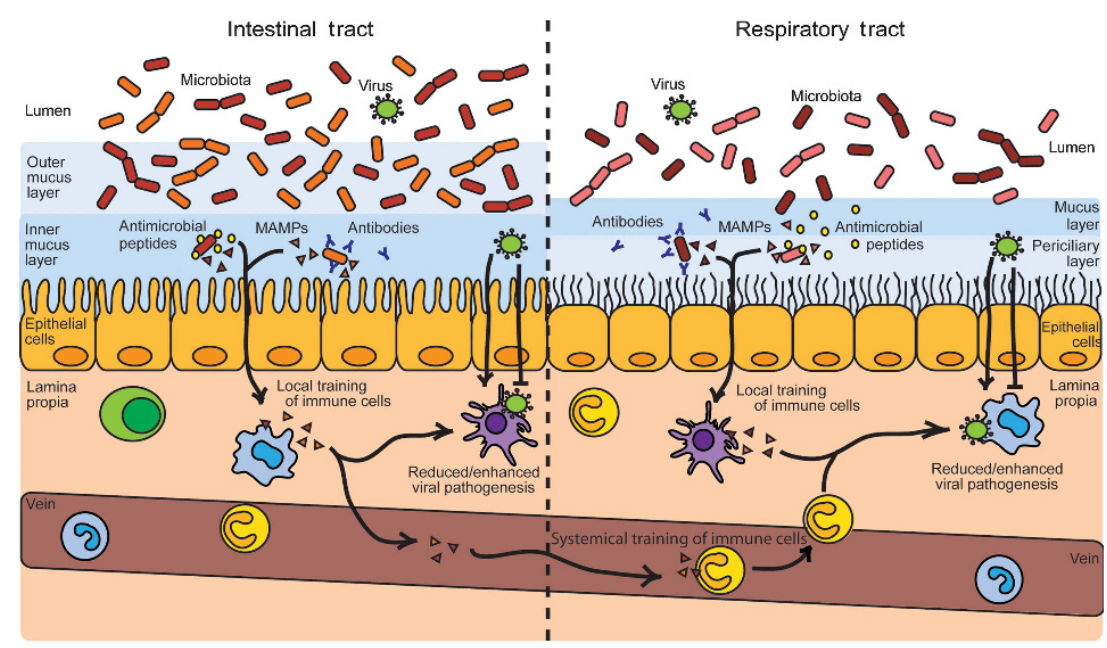

Figure 1 Bacteria and bacterial ligands can train immune cells both locally and systemically. The intestines have a very high diversity and abundance of commensals. Bacteria that invade the inner mucus layer are attacked by antimicrobial peptides and antibodies. Bacterial components are being released and these components can enter the lamina propia where they can locally train the immune cells or enter the bloodstream and train immune cells systemically. These systemically trained immune cells can enter other parts of the human body, e.g., the upper respiratory tract mucosa. The respiratory tract is also colonized by commensals and local training occurs in the lamina propia of the respiratory tract. The local or systemical training of immune cells can enhance or reduce viral pathogenesis, depending on the specific virus and bacteria involved. MAMP, microbe-associated molecular pattern. 


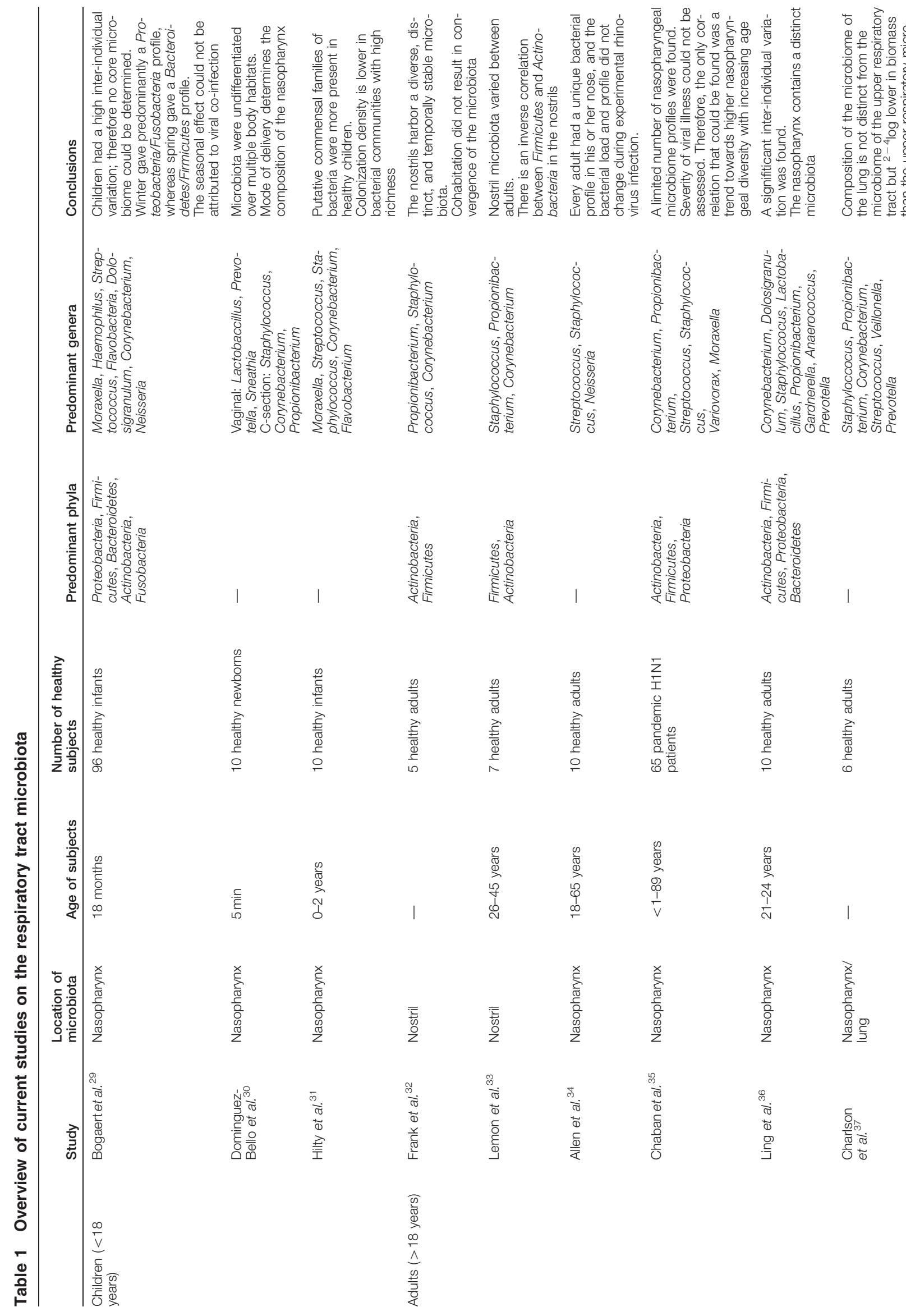




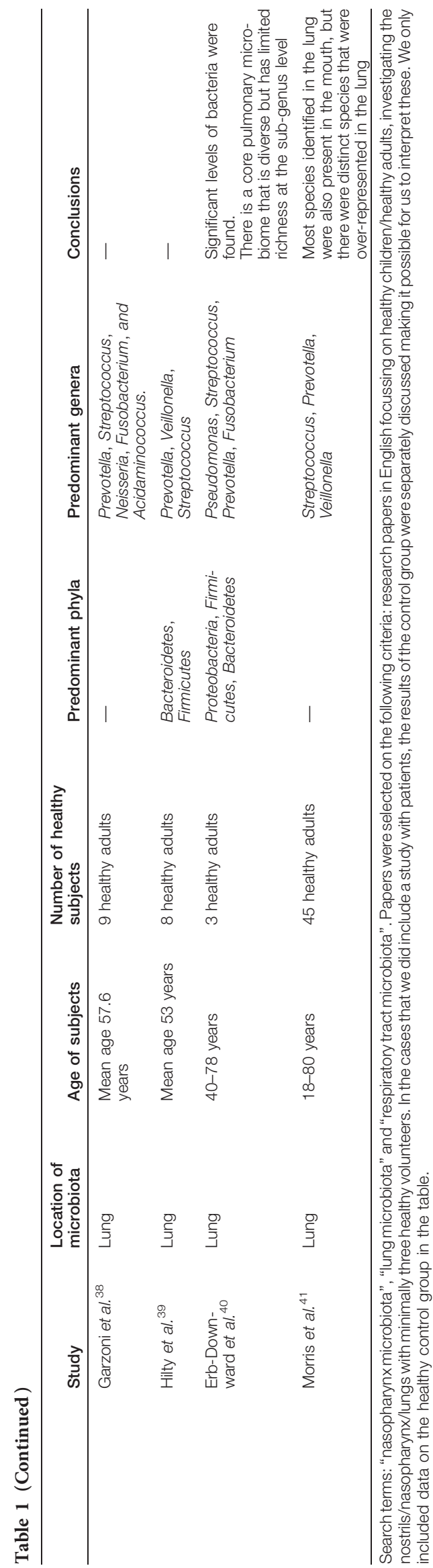

phylum level, mainly Firmicutes, Proteobacteria, and Bacteroidetes, and on species level, these are mainly Streptococcus, Neisseria, Haemophilus, and Lachnospira. ${ }^{33,37}$ The nasopharynx contains a combination of the skin lineages found on the nostrils (Firmicutes and Actinobacteria) combined with lineages typically found in the oral cavity (Proteobacteria and Bacteroidetes), e.g., Streptococcus, Staphylococcus, Corynebacterium, Neisseria, and Prevotella. ${ }^{34,36,37}$

Determination of the microbiota of the lower respiratory tract is complicated due to technical difficulties. Sampling of healthy donors without contaminating these samples with upper respiratory bacteria is difficult. Moreover, studies performed so far often focussed on individuals with lung disease, e.g., chronic obstructive pulmonary disorder, cystic fibrosis, and asthma, and mostly included small patients groups. Recently, several extensive reviews have been written on the role of the respiratory microbiome in disease. ${ }^{42,43}$ Studies have shown that the lungs of diseased and healthy patients show major differences. Healthy lungs contain low amounts of biomass thereby making it hard to detect the microbiota, whereas diseased lungs mostly contain measurable quantities of pathogens typically associated with the disease. ${ }^{44} \mathrm{~A}$ "core airway microbiota" has been described in healthy subjects, containing predominantly Pseudomonas, Streptococcus, Prevotella, Fusobacterium, Haemophilus, Veillonella, and Porphyromonas. ${ }^{40}$ However, other studies have been more sceptical. One study using healthy donors showed no clear distinction between microbiota of the upper and lower airways. Lung and oropharyngeal microbiota cluster together, separately from the nasopharyngeal microbiota. ${ }^{37}$ This may suggest that the bacteria that are found are the result of microaspiration of the microbiome of the upper respiratory tract. ${ }^{45}$ Current sequencing techniques do not enable a distinction between live and dead bacteria, which induces a significant bias. ${ }^{46}$ The question whether lungs contain a real separate microbiome is yet unanswered.

The influence of airway microbiota on the immune system The development of GF mice has provided an enormous advantage in the study of the interactions between microbiota and the immune system. However, GF mice lack commensal bacteria in general. Therefore, only the absence of all commensal bacteria can be studied, and no conclusions can be drawn about the lack of microbes at specific locations.

The presence of commensal bacteria is essential for normal cellular maturation, recruitment, and control of airway inflammation. ${ }^{47}$ Allergic GF mice have a stronger eosinophil influx, resulting in an increase of Th2 cytokines and thereby show an exaggerated allergic airway response. ${ }^{48}$ It has also been shown that GF mice have fewer IgM-producing B cells and $\mathrm{CD} 4+\mathrm{T}$ cells in the upper airways compared with the conventional mice. ${ }^{49}$ Another consequence of the modulation of microbiota can be the lymphocyte response towards bacterial RTI. The presence of commensal bacteria maintained the mucosal immune response towards Mycoplasma pulmonis infection. ${ }^{47}$ 
In contrast to the gut, not much is known about the influence of the nasopharyngeal microbiota on the local immune system. Larsen et al. ${ }^{50}$ stimulated monocyte-derived dendritic cells (DCs) with selected airway commensal bacteria (e.g., Viellonella), pathogenic bacteria (e.g., Haemophilus and Moraxella), and bacteria present in both healthy and sick lungs (e.g., Actinomyces). All bacteria activated DCs to a comparable level based on the surface expression of CD83, CD86, and CD40. However, pathogenic bacteria induced a 3-5-fold greater production of interleukin (IL)-23, IL-10, and IL-12p70. Coculture experiments showed that Prevotella reduced IL-12 production by Haemophilus by $50 \%$.

The development of GF mice has proven to be pivotal in studying interactions between commensals and the immune system. So far, to our knowledge, no studies have been performed that recolonized only the respiratory tract. This would enable us to study the effect of specific airway commensals on the development of the respiratory immune system.

\section{THE MICROBIOME HAS AN INFLUENCE ON SEVERITY OF VIRAL INFECTIONS}

As stated above, the presence of the microbiota is crucial for the development and maintenance of the human immune system. At the same time, the state of the immune system is important for the susceptibility towards viral infections. Commensal bacteria may either inhibit or enhance viral infections in direct or indirect ways.

\section{Gut commensals directly enhance local enteric virus infections}

Recently, two studies have shown that commensal bacteria in the gut can enhance enteric viral infections in a direct manner. ${ }^{51,52}$ Kane et al..$^{52}$ showed that mouse mammary tumor virus, a virus that is transmitted from mother to young through milk and invades through the gut, covers itself with lipopolysaccharide (LPS) from the commensal bacteria present in the gut. This virus-LPS complex is able to stimulate Toll-like receptor 4 (TLR4), which induces IL-6, which subsequently induces IL-10. The LPS-covered virus infects the cells, but due to the IL-10 production the antiviral response is shut off. Antibiotics kills these gut bacteria and thereby prevents viral infection and transmission of the virus. ${ }^{52}$ Kuss et al. ${ }^{51}$ showed that multiple viruses use ligands from the commensal bacteria to enhance their infection. Both poliovirus and reovirus are able to bind LPS and enhance attachment to their target cells and infect them. They showed that this enhanced infection was due to $\mathrm{N}$-acetylglucosamine-containing surface polysaccharides. ${ }^{51}$

\section{Gut commensals protect against systemic and respiratory viral infections}

As there have been millions of years of co-evolution between enteric viruses, commensal gut bacteria, and the hosts intestines, it is not surprising to find viruses that use commensal bacteria to aid their pathogenesis. What might be more striking is that the gut microbiota also has a systemic influence on viral infections.
Splenic DCs from GF mice are inhibited in their type I interferon (IFN) production and thus are not able to prime and activate natural killer (NK) cells. ${ }^{53}$ As a consequence, their antiviral immunity is severely compromised. Another study also showed that antibiotic treatment of mice led to decreased type I IFN expression and thereby a reduction in the expression of antiviral genes. ${ }^{54}$ This resulted in delayed viral clearance after a systemic infection (lymphocytic choriomeningitis virus) and, interestingly, a respiratory infection (influenza). The investigators concluded that host microbiota provide a tonic immune stimulation that establishes the activation threshold of the innate immune system for optimal antiviral immunity.

The influence of the commensal gut microbiota on the occurrence of RTI has been shown before. Clinical studies indicate that probiotics do not influence the incidence of RTI but do reduce the severity of the symptoms and the duration of the illness. ${ }^{55,56}$ In mice, the intake of Lactobacillus plantarum enhances the type I IFN response after influenza infection and thereby lowers viral titers in the lungs. ${ }^{57}$ Other Lactobacillus strains can enhance tumor necrosis factor $\alpha$ and IFN- $\gamma$ production by nasal lymphocytes to influenza infection. ${ }^{58}$

A recently published study indicates that mice given antibiotics have a disturbance in their gut microbiota. Lower influenza-specific antibody titers and lower $\mathrm{CD} 4+$ and CD8 + T-cell responses result in higher viral titers in the lungs of these mice. ${ }^{59}$ Moreover, it was shown that both intranasal and intrarectal administration of LPS repairs this immune impairment. This mechanism may be a two-hit model in which the intact microbiota first provide signals, e.g., LPS, which lead to pro-IL-1 $\beta$ and pro-IL-18 expression, and secondly, the influenza infection induces the inflammasome, which then converts the pro-forms into IL- $1 \beta$ and IL-18. DCs are then directed towards the lymph nodes and are able to prime the $\mathrm{T}$ cells present there. The effect of the commensal gut microbiota towards RTI can also be indirect. Tanaka et al. ${ }^{60}$ showed that indigenous microbiota of mice maintained the mouse cytomegalovirus (MCMV)-specific CD8 + memory cells in the lungs, probably due to cross-reactivity of the antigenic epitope of MCMV T cells and the enormous variety of peptides in the microbiota. Whether this would be beneficial (faster clearance of MCMV upon reinfection) or possibly detrimental (enhanced immunopathogenesis upon reinfection) is not known.

\section{Respiratory tract microbiota could have a dual role in viral infections}

Comparable to the intestinal tract, the commensals, viruses, and host respiratory tract also have a long history of co-evolution, and it may be expected that certain viruses make use of the present commensal bacteria to facilitate infection. Literature on the influence of the specific respiratory tract microbiota on viral RTI is limited. Bacteria or bacterial ligands can either enhance or reduce viral infection rate (top part of Figure 2) or they can influence the subsequent immune response of the host towards a viral infection in either an enhancing or reducing way (bottom part of Figure 2). 


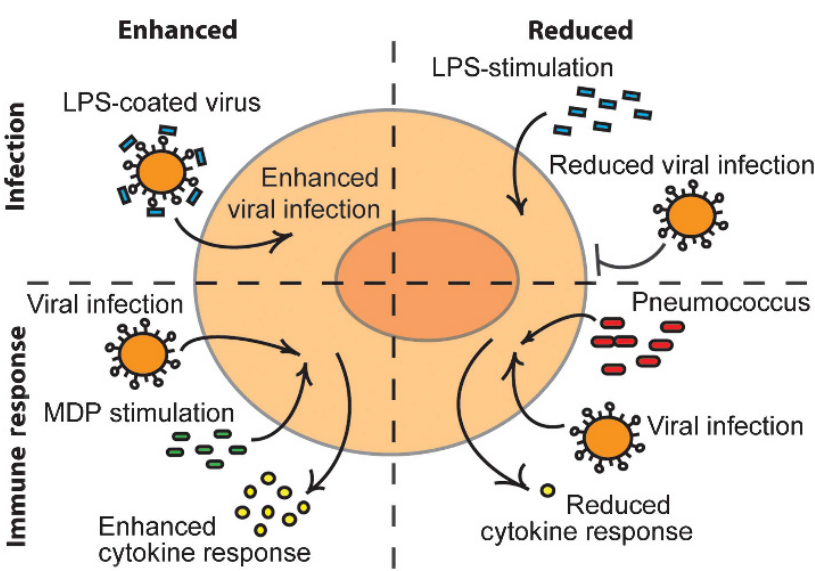

Figure 2 Bacteria or bacterial ligands can influence viral pathogenesis in multiple ways. They can influence viral infection rate itself, as can be seen in the upper part of the figure. Bacteria or bacterial components are able to enhance or inhibit viral infection rate, depending on the specific virus and ligands involved. It can also influence viral pathogenesis in an indirect manner. Most respiratory viruses induce immunopathogenesis. The lower part of the figure shows how bacteria or its components can influence the subsequent immune response of the host towards the viral infection, thereby influencing the pathogenesis. LPS, lipopolysaccharide; MDP, muramyl dipeptide.

As discussed in the section on the influence of intestinal microbiota on enteric viruses, certain viruses are able to coat themselves with LPS thereby enhancing their infection rates. ${ }^{51,52}$ However, at this point this has not been described for respiratory viruses. Viral replication in the respiratory tract can be enhanced by Staphylococcal enterotoxins or exposure to S. pneumoniae. ${ }^{61,62}$ One study showed that LPS could also reduce viral infection rates. In vitro pre-stimulation of human macrophages with LPS induced an antiviral response, which reduced RSV and influenza infection by $80 \% .{ }^{63}$ LPS seems to be a ligand that can influence viral pathogenesis in multiple ways. Therefore, the receptor for LPS, TLR4, is of interest. TLR4 has also been described as being a receptor that is able to recognize the F-protein of RSV. ${ }^{64}$ Whether or not this dual function of TLR4 is also of importance for the interaction between commensal bacteria and viral infections is not known.

In vitro studies in human primary cells have shown that the pro-inflammatory response to a viral infection can be enhanced by a specific bacterial ligand, muramyl dipeptide (MDP). ${ }^{65}$ Multiple respiratory viruses, including RSV, are able to induce type I IFNs, upregulating the receptor for MDP, namely NOD2 (nucleotide-binding oligomerization domain-containing protein 2). Subsequent stimulation by MDP leads to a severely enhanced pro-inflammatory response, which could potentially facilitate the immunopathogenesis of RSV infection.

Respiratory commensal bacteria can also reduce the immune response to a viral infection. The presence of a commensal nasopharyngeal microbiota protected mice against RSVinduced airway hyper-responsiveness. Mice were infected with RSV and subsequently treated with antibiotics, which depleted the nasopharynx from Streptococcus viridans. This led to increased numbers of inflammatory lymphocytes, decreased $\mathrm{T}_{\text {regs }}$ and transforming growth factor- $\beta$ production and enhanced airway hyper-responsiveness. This effect was limited within local tissues and not systemic. ${ }^{66}$ Influenza-infected mice showed an enhanced recruitment of inflammatory monocytes to the lungs when they were stimulated with MDP. This resulted in a reduced pulmonary inflammation, viral load, and mortality. ${ }^{67}$

Pre-stimulation of cultured human airway epithelial cells with Haemophilus influenzae induced an upregulation of intercellular adhesion molecule- 1 and TLR3. This resulted in an increased binding of rhinovirus and a subsequent stronger IL-8 response ${ }^{68}$ This study shows that certain pathogens can induce a stronger infection as well as an enhanced immune response.

Although indirect, there is also some evidence from clinical studies. Some studies have shown that the effect of the pneumococcal conjugate vaccine is broader than just a reduction in pneumococcal carriage and infection. ${ }^{69,70}$ The reduction in pneumococcal carriage and infection also results in a reduction of $31 \%$ in viral RTI. Individuals with acute viral infections are more often and more heavily colonized by specific bacteria. ${ }^{71-73}$ Therefore, it can be argued that colonization with S. pneumoniae increases the risk to get infected with respiratory viruses or that its presence enhances symptoms. However, it cannot be excluded that viral infection also affects colonization rate and spread of pneumococcus.

So far, literature clearly shows that commensal microbiota affect our susceptibility to viral infections. An overview on current studies on the influence of the respiratory microbiota on respiratory viral infections can be found in Table 2. This effect can either be indirect, by modulating the immune system, or direct, e.g., viruses that use bacterial ligands to enhance their infection (Figure 2). Current evidence suggests that the influence of the gut microbiota on systemic immunity is mostly protective against viral infections, whereas local commensal bacteria and local immune responses, both in the intestines and in the respiratory tract, can either enhance or eliminate the viral infection.

\section{THE FORMATION AND IMPLICATIONS OF THE NEONATAL MICROBIOTA}

As shown above, it is well accepted that the presence of commensal microbiota has a major influence on the training of the immune system. Moreover, we provided an overview of the literature demonstrating the influence of this commensal microbiota on viral infections. However, viral RTI pose a big disease burden to very young infants. In this part of the review, we will summarize the literature regarding the initial colonization and how this influences the developing immune system.

\section{The microbiome of infants is subject to enormous changes} During and immediately after birth, the newborn becomes colonized. Newborns receive their first colonizers from their passage through the mother's vaginal tract, contact with their surroundings, nurses, parents, visitors, and by feeding. 


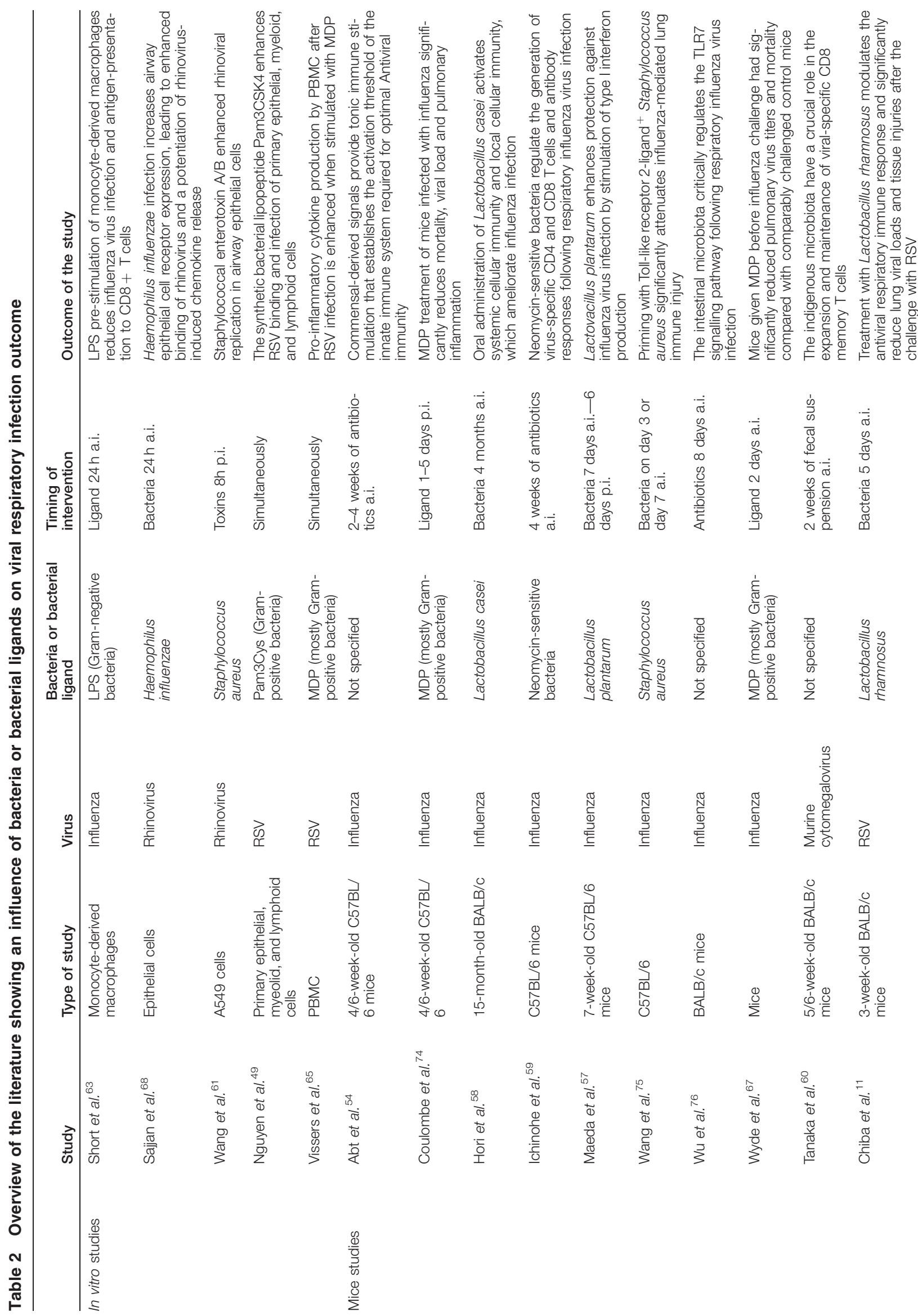




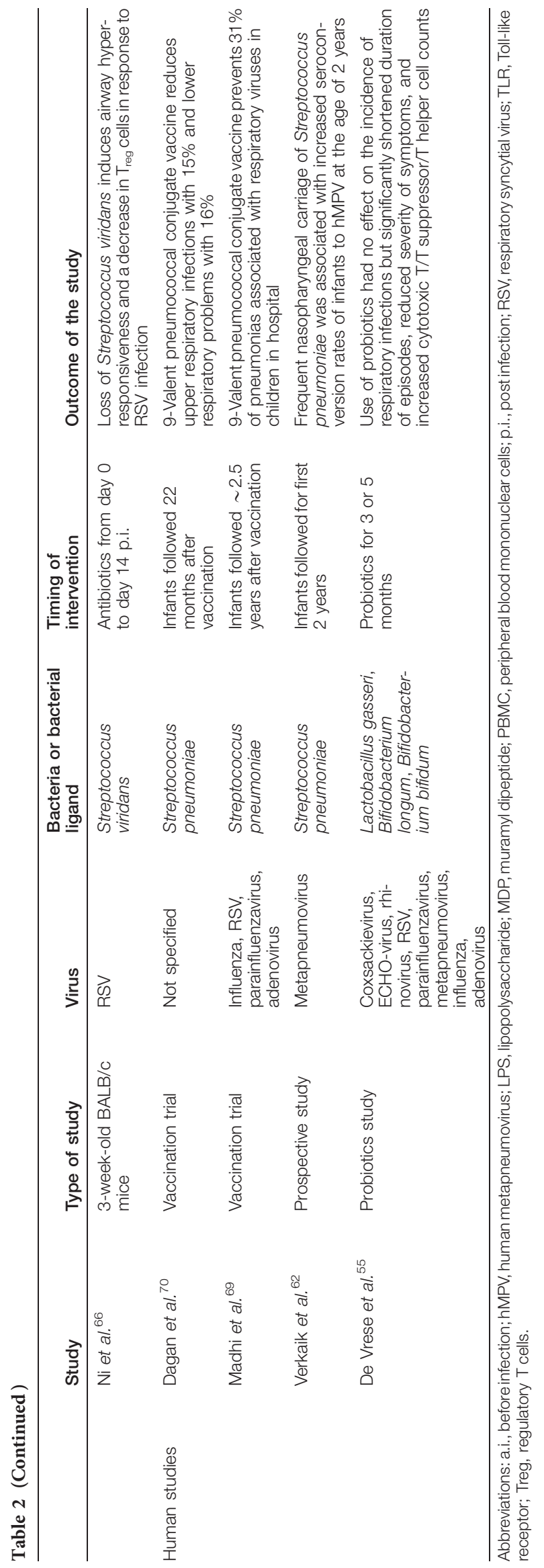

Colonization is influenced by multiple factors, such as age, ${ }^{77}$ mode of delivery, ${ }^{30}$ breastfeeding, ${ }^{78}$ antibiotic use, ${ }^{79}$ vaccination, ${ }^{80}$ season, ${ }^{29}$ exposure to smoke, ${ }^{81}$ day-care attendance, ${ }^{82,83}$ and presence of siblings. ${ }^{84}$

Initial colonizers of the gastrointestinal tract are mainly facultative anaerobes (e.g., Lactobacilli and Enterobacteria), subsequently obligate anaerobes will follow quickly (e.g., Bacteroides and Bifidobacterium). Over time, the microbiota increases in diversity and stability and mirrors the adult pattern by the age of $1-3$ years. ${ }^{77,85}$

An important determinant of the initial colonizers is the mode of delivery. Newborns who are vaginally delivered have microbiota that resemble the vaginal microbiota (Lactobacillus, Prevotella) of their mother, while newborns delivered by C-section have microbiota resembling the mother's skin (Staphylococcus, Corynebacterium). ${ }^{30}$ In contrast to adults, the microbiota of newborns are undifferentiated across multiple body habitats, thereby showing that only a subset of these initial colonizers will permanently colonize the infant and will become a part of the distinctive microbiota across multiple body habitats. Several studies have shown that the initial colonizers are important as newborns delivered by $\mathrm{C}$-section are more susceptible to certain pathogens, ${ }^{86}$ which may be the result of a delayed colonization by Lactobacillus, Bifidobacterium, and Bacteroides. ${ }^{87,88}$

Other components also influence the colonization in the first months, e.g., breast-feeding and birth weight. Newborns with extremely low birth weights have less Bacteroidetes. ${ }^{89}$ Infants that are breast-fed show a higher diversity in microbiota and have more Bacteroidetes, ${ }^{78}$ which are associated with a better health and less allergies later in life. ${ }^{90}$

Hansen et al. ${ }^{91}$ have shown in mice that there is a time window in which young mice can be efficiently colonized. Inoculation of GF mice at 3 weeks of age changed their microbiota composition to the bacteria that were inoculated. However, GF mice at 1 week of age that were inoculated developed a microbiota, which was comparable to specific pathogen-free mice.

Other investigators studied the time-dependent development of the microbiota in different organ systems. ${ }^{92}$ They showed that certain clusters of bacteria were first present in the gut, before colonizing the respiratory tract. This highlights the interaction between these two organ systems and their microbiota. A different diet induced different intestinal microbiota and subsequently different respiratory microbiota.

Not many studies have focused on the upper respiratory tract microbiota in healthy children (Table 1). Bogaert et al. ${ }^{29}$ showed that the most pre-dominant phyla of the nasopharynx of young children are Proteobacteria, Firmicutes, Bacteroidetes, Actinobacteria, and Fusobacteria. They showed that there were distinct microbiota during fall/winter (mostly Proteobacteria and Fusobacteria) and spring (mostly Bacteroidetes and Firmicutes). The majority of the children had a predominant Gram-negative nasopharyngeal community profile, which was even stronger in winter. They hypothesized that the springmicrobiota is more balanced and thereby protective against overgrowth of pathogenic species. 


\section{The initial colonizing microbiome is crucial for the development of the immune system}

Certain parts of the innate and adaptive immune system of infants are still immature after birth (extensively reviewed by Martin et $a .^{93}$ ). The development of a stable microbiome occurs therefore during a time that corresponds to a critical period of immune development and maturation. While a fetus is in the womb, pro-inflammatory responses are suppressed so as to avoid adverse immunological reactions between mother and child. Children are therefore born with immunological tolerance, making it possible to be colonized by bacteria.

The introduction of GF mice in research clearly shows how important commensal bacteria are for the development of the immune system. GF mice have poorly developed gut-associated lymphoid tissue, less intraepithelial lymphocytes, smaller Peyer's patches, hardly any mature isolated lymphoid follicles and low IgA levels. ${ }^{93}$

Mice studies have shown that a short GF period after birth changes the numbers of $\mathrm{T}_{\text {regs }}, \mathrm{NK}$, and NKT cells and cytokine levels permanently. ${ }^{91}$ In contrast to adult mice, colonization of neonatal GF mice protected them from accumulation of NKT cells in the colonic lamina propia and the lungs. This resulted in a decreased pathology when inflammatory bowel disease or allergic asthma was induced. ${ }^{94}$ So a small time window exists in which one can be efficiently colonized, which will modify future immune responses and pathology.

A study in infants showed that breast-fed infants have gut microbiota that are richer in virulence genes, and this is correlated with upregulation of immunity-related genes. ${ }^{78}$ Another study showed that infants who were early and intensely colonized by Bacteroides fragilis had a downregulated LPS responsiveness. ${ }^{95}$

Strachan ${ }^{96}$ was the first to publish that hay fever and eczema (both allergic diseases) were less common in children from larger families. This resulted in the formulation of the "hygiene hypothesis", which poses that a lack of early childhood exposure to infectious agents and symbiotic microorganisms increases susceptibility to allergic diseases, even at distant locations like, e.g., the skin or the upper airways, by suppressing the natural development of the immune system. Epidemiological studies have shown a correlation between the prevalence of asthma and airway allergies and variations in the gastrointestinal tract bacteria, e.g., lower amounts of Lactobacilli and Bifidobacteria. ${ }^{97}$ Several reviews on the use of probiotics in humans to prevent respiratory infections or allergic airway disease show that studies so far have given rise to conflicting evidence. $^{98,99}$ The majority of the reviewed studies have shown a beneficial effect on respiratory infections or allergic airway disease. ${ }^{100-105}$ However, there are also studies that show no difference. ${ }^{106,107}$ One study performed in mice even showed a more severe allergic airway response after neonatal treatment of mice with Lactobacillus casei. ${ }^{108}$ Most probiotic trials have tried to reduce respiratory burden via oral intake of probiotics. ${ }^{58,109}$ However, some studies have shown that nasal administration of probiotics might be more efficient. ${ }^{110,111}$
There is a lack of data on the development of the microbiome and the immune system in young infants. However, it is clear that the initial colonization is crucial for the development of the immune system. In the future, it will be crucial to study the initial colonization and immune development of infants in more detail.

\section{CONCLUDING REMARKS AND FUTURE DIRECTIONS}

The influence of the intestinal microbiome on the immune system is widely accepted. Evidence suggests that the intestinal microbiome has an influence on systemic immunity and immunity in distant locations, such as the respiratory tract. However, the respiratory tract by itself is also colonized with commensal bacteria, and it is to be expected that these commensals also influence the local respiratory immunity. A growing body of evidence suggests that microbiota influence viral infections in several ways. This influence can be either detrimental or beneficial for viral infections. Again, the basis for this evidence is stronger for intestinal microbiota as compared with that for the respiratory tract. However, by comparison with the intestinal tract one would assume that the respiratory microbiota influences viral RTI. They could enable or prevent direct virus infection, prime the immune system for a viral infection, or perhaps enhance the immune response once a virus has infected epithelial cells. For multiple respiratory viruses, part of the disease pathogenesis is an enhanced immune/inflammatory response. Animal models should be developed to specifically study the influence of the nasopharyngeal microbiota both on local immune development as well as the severity of RTI. Whether the influence comes from functional bacterial groups or perhaps "keystone species"112 is not known and could be different for each type of viral infection. Research into which specific bacteria are involved will be crucial for future understanding.

Timing and composition of the initial colonization in infants has lifelong consequences for the immune response. The development of the immature immune system happens simultaneously with the quickly changing composition of the microbiota. Therefore, one can imagine that the initial colonization and also the presence of specific bacterial strains during a viral RTI influences the disease severity. Studies in infants have shown that not only pneumococcal vaccination but also the use of probiotics can protect against severe viral RTI. Consequently, host microbiota can no longer be ignored when studying host-viral interactions. A more thorough understanding of the ontogeny of the microbiome in infants is critically required.

Advances in both sequencing technologies and the development of important mice models herald a new era in characterizing the role of the microbiota in the severity of infections. Comprehension of the impact of the microbiota on the susceptibility to severe RTI, together with a better understanding of the dynamics and kinetics of colonization during infancy, will allow new possibilities for the treatment and detection of highly susceptible infants. 


\section{ACKNOWLEDGMENTS}

We are very grateful to Professor Octavio Ramilo and to Dr Hester Bootsma for their critical comments on the manuscript. M.V. and G.F. are financially supported by the VIRGO consortium, funded by the Dutch government, project number FES0908, and by the Netherlands Genomics Initiative (NGI), project number 050-060-452. The funders had no role in the decision to publish or preparation of the manuscript.

\section{DISCLOSURE}

The authors declared no conflict of interest.

c) 2014 Society for Mucosal Immunology

\section{REFERENCES}

1. Kieny, M.P. \& Girard, M.P. Human vaccine research and development: an overview. Vaccine 23, 5705-5707 (2005).

2. Mizgerd, J.P. Lung infection—a public health priority. PLoS Med. 3, e76 (2006).

3. Hall, C.B. et al. The burden of respiratory syncytial virus infection in young children. N. Engl. J. Med. 360, 588-598 (2009).

4. Sly, P.D. et al. Role of respiratory viruses in acute upper and lower respiratory tract illness in the first year of life: a birth cohort study. Pediatr. Infect. Dis. J. 25, 680-686 (2006).

5. Canducci, F. et al. Two-year prospective study of single infections and coinfections by respiratory syncytial virus and viruses identified recently in infants with acute respiratory disease. J. Med. Virol. 80, 716-723 (2008).

6. Brand, H.K. et al. Infection with multiple viruses is not associated with increased disease severity in children with bronchiolitis. Pediatr. Pulmonol. 47, 393-400 (2012).

7. Simoes, E. Environmental and demographic risk factors for respiratory syncytial virus lower respiratory tract disease. J. Pediatr. 143 (5 Suppl), S118 (2003).

8. Wang, E.E., Law, B.J. \& Stephens, D. Pediatric Investigators Collaborative Network on Infections in Canada (PICNIC) prospective study of risk factors and outcomes in patients hospitalized with respiratory syncytial viral lower respiratory tract infection. J. Pediatr. 126, 212-219 (1995).

9. Tregoning, J.S. \& Schwarze, J. Respiratory viral infections in infants: causes, clinical symptoms, virology, and immunology. Clin. Microbiol. Rev. 23, 74-98 (2010).

10. Boyce, T.G., Mellen, B.G., Mitchel, E. Jr, Wright, P.F. \& Griffin, M.R. Rates of hospitalization for respiratory syncytial virus infection among children in medicaid. J. Pediatr. 137, 865 (2000).

11. Chiba, E. et al. Immunobiotic Lactobacillus rhamnosus improves resistance of infant mice against respiratory syncytial virus infection. Int. Immunopharmacol. 17, 373-382 (2013).

12. Hooper, L.V., Midtvedt, T. \& Gordon, J.I. How host-microbial interactions shape the nutrient environment of the mammalian intestine. Annu. Rev. Nutr. 22, 283-307 (2002).

13. Bäckhed, F., Ley, R.E., Sonnenburg, J.L., Peterson, D.A. \& Gordon, J.I. Host-bacterial mutualism in the human intestine. Science 307 , 1915-1920 (2005)

14. Ley, R.E., Peterson, D.A. \& Gordon, J.I. Ecological and evolutionary forces shaping microbial diversity in the human intestine. Cell 124, 837-848 (2006).

15. Honda, K. \& Littman, D.R. The microbiome in infectious disease and inflammation. Annu. Rev. Immunol. 30, 759-795 (2012).

16. Turnbaugh, P.J. et al. An obesity-associated gut microbiome with increased capacity for energy harvest. Nature 444, 1027-1131 (2006).

17. Clemente, J.C., Ursell, L.K., Parfrey, L.W. \& Knight, R. The impact of the gut microbiota on human health: an integrative view. Cell 148, 1258-1270 (2012).

18. Goldszmid, R.S. \& Trinchieri, G. The price of immunity. Nat. Immunol. 13, 932-938 (2012).

19. Hooper, L.V., Littman, D.R. \& Macpherson, A.J. Interactions between the microbiota and the immune system. Science 336, 1268-1273 (2012).

20. Chinen, T. \& Rudensky, A.Y. The effects of commensal microbiota on immune cell subsets and inflammatory responses. Immunol. Rev. 245, 45-55 (2012).
21. Maynard, C.L., Elson, C.O., Hatton, R.D. \& Weaver, C.T. Reciprocal interactions of the intestinal microbiota and immune system. Nature 489 , 231-241 (2012).

22. Clarke, T.B. et al. Recognition of peptidoglycan from the microbiota by Nod1 enhances systemic innate immunity. Nat. Med. 16, 228-231 (2010).

23. Lee, Y.K., Menezes, J.S., Umesaki, Y. \& Mazmanian, S.K. Proinflammatory T-cell responses to gut microbiota promote experimental autoimmune encephalomyelitis. Proc. Natl. Acad. Sci. 108 (Supplement 1), 4615-4622 (2011).

24. Wu, H.-J. et al. Gut-residing segmented filamentous bacteria drive autoimmune arthritis via T helper 17 cells. Immunity 32, 815-827 (2010).

25. Atarashi, $\mathrm{K}$. et al. Induction of colonic regulatory $\mathrm{T}$ cells by indigenous Clostridium species. Sci. Signal. 331, 337 (2011).

26. Mazmanian, S.K., Liu, C.H., Tzianabos, A.O. \& Kasper, D.L. An immunomodulatory molecule of symbiotic bacteria directs maturation of the host immune system. Cell 122, 107-118 (2005).

27. Noverr, M.C. \& Huffnagle, G.B. Does the microbiota regulate immune responses outside the gut? Trends Microbiol. 12, 562-568 (2004).

28. Oyama, N., Sudo, N., Sogawa, H. \& Kubo, C. Antibiotic use during infancy promotes a shift in the $\mathrm{T}_{\mathrm{H} 1} / \mathrm{T}_{\mathrm{H} 2}$ balance toward $\mathrm{T}_{\mathrm{H} 2}$-dominant immunity in mice. J. Allergy Clin. Immunol. 107, 153-159 (2001).

29. Bogaert, D. et al. Variability and diversity of nasopharyngeal microbiota in children: a metagenomic analysis. PLoS One 6, e17035 (2011).

30. Dominguez-Bello, M.G. et al. Delivery mode shapes the acquisition and structure of the initial microbiota across multiple body habitats in newborns. Proc. Natl. Acad. Sci. 107, 11971-11975 (2010).

31. Hilty, M. et al. Nasopharyngeal microbiota in infants with acute otitis media. J. Infect. Dis. 205, 1048-1055 (2012).

32. Frank, D.N. et al. The human nasal microbiota and Staphylococcus aureus carriage. PLoS One 5, e10598 (2010).

33. Lemon, K.P. et al. Comparative analyses of the bacterial microbiota of the human nostril and oropharynx. MBio 1, e00129-10 (2010).

34. Allen, E.K. et al. Bacteria in the nose of young adults during wellness and rhinovirus colds: detection by culture and microarray methods in 100 nasal lavage specimens. Proc. Int. Forum Allergy Rhinol., Wiley Online Library 3, 731-739 (2013).

35. Chaban, B. et al. Characterization of the upper respiratory tract microbiomes of patients with pandemic H1N1 influenza. PloS One 8, e69559 (2013).

36. Ling, Z. et al. Pyrosequencing analysis of the human microbiota of healthy Chinese undergraduates. BMC Genomics 14, 390 (2013).

37. Charlson, E.S. et al. Topographical continuity of bacterial populations in the healthy human respiratory tract. Am. J. Respir. Crit. Care Med. 184, 957-963 (2011).

38. Garzoni, C. et al. Microbial communities in the respiratory tract of patients with interstitial lung disease. Thorax; doi:10.1136/thoraxjnl-2012-202917 (e-pub ahead of print) (2013).

39. Hilty, M. et al. Disordered microbial communities in asthmatic airways. Plos One 5, e8578 (2010).

40. Erb-Downward, J.R. et al. Analysis of the lung microbiome in the "healthy" smoker and in COPD. PloS One 6, e16384 (2011).

41. Morris, A. et al. Comparison of the respiratory microbiome in healthy nonsmokers and smokers. Am. J. Respir. Crit. Care Med. 187, 1067-1075 (2013).

42. Gollwitzer, E.S. \& Marsland, B.J. Microbiota abnormalities in inflammatory airway diseases-potential for therapy. Pharmacol. Ther.; doi:10.1016/ j.pharmthera.2013.08.002 (e-pub ahead of print) (2013).

43. Huang, Y.J. et al. The role of the lung microbiome in health and disease: a National Heart, Lung and Blood Institute Workshop Report. Am. J. Respir. Crit. Care Med. 187, 1382-1387 (2013). (ja).

44. Goddard, A.F. et al. Direct sampling of cystic fibrosis lungs indicates that DNA-based analyses of upper-airway specimens can misrepresent lung microbiota. Proc. Natl. Acad. Sci. 109, 13769-13774 (2012).

45. Beck, J.M., Young, V.B. \& Huffnagle, G.B. The microbiome of the lung. Transl. Res. 160, 258-266 (2012).

46. Rogers, G.B. et al. Assessing the diagnostic importance of nonviable bacterial cells in respiratory infections. Diagn. Microbiol. Infect. Dis. 62, 133-141 (2008).

47. Henriksson, G., Helgeland, L., Midtvedt, T., Stierna, P. \& Brandtzaeg, P. Immune response to Mycoplasma pulmonis in nasal mucosa is 
modulated by the normal microbiota. Am. J. Respir. Cell Mol. Biol. 31, 657-662 (2004).

48. Herbst, T. et al. Dysregulation of allergic airway inflammation in the absence of microbial colonization. Am. J. Respir. Crit. Care Med. 184, 198-205 (2011).

49. Nguyen, D.T. et al. The synthetic bacterial lipopeptide Pam3CSK4 modulates respiratory syncytial virus infection independent of TLR activation. PLoS Pathogens 6, e1001049 (2010).

50. Larsen, J.M. et al. Divergent pro-inflammatory profile of human dendritic cells in response to commensal and pathogenic bacteria associated with the airway microbiota. PLoS One 7, e31976 (2012).

51. Kuss, S.K. et al. Intestinal microbiota promote enteric virus replication and systemic pathogenesis. Sci. Signal. 334, 249 (2011).

52. Kane, M. et al. Successful transmission of a retrovirus depends on the commensal microbiota. Sci. Signal. 334, 245 (2011).

53. Ganal, S.C. et al. Priming of natural killer cells by nonmucosal mononuclear phagocytes requires instructive signals from commensal microbiota. Immunity 37, 171-186 (2012).

54. Abt, M.C. et al. Commensal bacteria calibrate the activation threshold of innate antiviral immunity. Immunity 37, 158-170 (2012).

55. DeVrese, M. etal. Probiotic bacteria reduced duration and severity but not the incidence of common cold episodes in a double blind, randomized, controlled trial. Vaccine 24, 6670-6674 (2006).

56. Vouloumanou, E.K., Makris, G.C., Karageorgopoulos, D.E. \& Falagas, M.E. Probiotics for the prevention of respiratory tract infections: a systematic review. Int. J. Antimicrob. Agents 34197, e1-10 (2009).

57. Maeda, N. et al. Oral administration of heat-killed Lactobacillus plantarum $\mathrm{L}-137$ enhances protection against influenza virus infection by stimulation of type I interferon production in mice. Int. Immunopharmacol. 9, 1122-1125 (2009).

58. Hori, T., Kiyoshima, J., Shida, K. \& Yasui, H. Augmentation of cellular immunity and reduction of influenza virus titer in aged mice fed Lactobacillus casei strain Shirota. Clin. Diagn. Lab. Immunol. 9, 105-108 (2002).

59. Ichinohe, $\mathrm{T}$. et al. Microbiota regulates immune defense against respiratory tract influenza A virus infection. Sci. Signal. 108, 5354 (2011).

60. Tanaka, K., Sawamura, S., Satoh, T., Kobayashi, K. \& Noda, S. Role of the indigenous microbiota in maintaining the virus-specific CD8 memory $T$ cells in the lung of mice infected with murine cytomegalovirus. J. Immunol. 178, 5209-5216 (2007).

61. Wang, J.H., Kwon, H.-J., Lee, B.-J. \& Jang, Y.J. Staphylococcal enterotoxins $A$ and $B$ enhance rhinovirus replication in A549 cells. Am. J. Rhinol. 21, 670-674 (2007).

62. Verkaik, N. et al. Streptococcus pneumoniae exposure is associated with human metapneumovirus seroconversion and increased susceptibility to in vitro HMPV infection. Clin. Microbiol. Infect. 17, 1840-1844 (2011).

63. Short, K.R. et al. Bacterial lipopolysaccharide inhibits influenza virus infection of human macrophages and the consequent induction of CD8 + T cell immunity. J. Innate Immun. (e-pub ahead of print) (2013).

64. Kurt-Jones, E.A. et al. Pattern recognition receptors TLR4 and CD14 mediate response to respiratory syncytial virus. Nat. Immunol. 1, 398-401 (2000).

65. Vissers, M. et al. Respiratory syncytial virus infection augments NOD2 signaling in an IFN- $\beta$-dependent manner in human primary cells. Eur. J. Immunol. 42, 2727-2735 (2012).

66. Ni, K. et al. Pharyngeal microflora disruption by antibiotics promotes airway hyperresponsiveness after respiratory syncytial virus infection. PLoS One 7, e41104 (2012).

67. Wyde, P.R., Six, H.R., Ambrose, M.W. \& Throop, B.J. Muramyl peptides and polyinosinic-polycytodylic acid given to mice prior to influenza virus challenge reduces pulmonary disease and mortality. J. Immunother. 9, 98-102 (1990).

68. Sajjan, U.S. et al. H. influenzae potentiates airway epithelial cell responses to rhinovirus by increasing ICAM-1 and TLR3 expression. FASEB J. 20, 2121-2123 (2006).

69. Madhi, S.A. \& Klugman, K.P. A role for Streptococcus pneumoniae in virus-associated pneumonia. Nat. Med. 10, 811-813 (2004).

70. Dagan, R. et al. Effect of a conjugate pneumococcal vaccine on the occurrence of respiratory infections and antibiotic use in day-care center attendees. Pediatr. Infect. Dis. J. 20, 951-958 (2001).
71. Burky, E. \& Smillie, W. Nasopharyngeal flora in health and during respiratory disease in isolated communities in Alabama and Labrador. J. Exp. Med. 50, 643-663 (1929).

72. Webster, L. \& Clow, A. The association of Pneumococci, Hemophilus influenzae, and Streptococcus hemolyticus with coryza, pharyngitis, and sinusitis in man. J. Exp. Med. 55, 445-453 (1932).

73. Wadowsky, R.M., Mietzner, S.M., Skoner, D.P., Doyle, W.J. \& Fireman, P. Effect of experimental influenza A virus infection on isolation of Streptococcus pneumoniae and other aerobic bacteria from the oropharynges of allergic and nonallergic adult subjects. Infect. Immun. 63, 1153-1157 (1995).

74. Coulombe, F., Fiola, S., Akira, S., Cormier, Y. \& Gosselin, J. Muramyl dipeptide induces NOD2-dependent Ly6Chigh monocyte recruitment to the lungs and protects against influenza virus infection. PLoS One 7, e36734 (2012).

75. Wang, J. et al. Bacterial colonization dampens influenza-mediated acute lung injury via induction of $\mathrm{M} 2$ alveolar macrophages. Nat. Commun. 4, 2106 (2013).

76. Wu, S. et al. Microbiota regulates the TLR7 signaling pathway against respiratory tract influenza a virus infection. Curr. Microbiol. 67, 414-422 (2013).

77. Yatsunenko, T. et al. Human gut microbiome viewed across age and geography. Nature 486, 222-227 (2012).

78. Schwartz, S. et al. A metagenomic study of diet-dependent interaction between gut microbiota and host in infants reveals differences in immune response. Genome Biol. 13, r32 (2012).

79. Dagan, R. et al. Dynamics of pneumococcal nasopharyngeal colonization during the first days of antibiotic treatment in pediatric patients. Pediatr. Infect. Dis. J. 17, 880-885 (1998).

80. Huang, S.S. et al. Continued impact of pneumococcal conjugate vaccine on carriage in young children. Pediatrics 124, e1-e11 (2009).

81. Murphy, T.F. Otitis media, bacterial colonization, and the smoking parent. Clin. Infect. Dis. 42, 904-906 (2006).

82. Farjo, R.S. et al. Diversity and sharing of Haemophilus influenzae strains colonizing healthy children attending day-care centers. Pediatr. Infect. Dis. J. 23, 41-46 (2004).

83. Yagupsky, P. et al. Acquisition, carriage, and transmission of pneumococci with decreased antibiotic susceptibility in young children attending a day care facility in southern Israel. J. Infect. Dis. 177, 1003-1012 (1998).

84. Givon-Lavi, N., Fraser, D., Porat, N. \& Dagan, R. Spread of Streptococcus pneumoniae and antibiotic-resistant S. pneumoniae from day-care center attendees to their younger siblings. J. Infect. Dis. 186, 1608-1614 (2002).

85. Palmer, C., Bik, E.M., Digiulio, D.B., Relman, D.A. \& Brown, P.O. Development of the human infant intestinal microbiota. PLoS Biol. 5, e177 (2007).

86. Watson, J. et al. Community-associated methicillin-resistant Staphylococcus aureus infection among healthy newborns-Chicago and Los Angeles County, 2004. JAMA 296, 36-38 (2006).

87. Adlerberth, I. et al. Reduced enterobacterial and increased staphylococcal colonization of the infantile bowel: an effect of hygienic lifestyle? Pediatr. Res. 59, 96-101 (2006).

88. Grölund, M.-M., Lehtonen, O.-P., Eerola, E. \& Kero, P. Fecal microflora in healthy infants born by different methods of delivery: permanent changes in intestinal flora after cesarean delivery. J. Pediatr. Gastroenterol. Nutr. 28, 19-25 (1999).

89. Latuga, M.S. et al. Beyond bacteria: a study of the enteric microbial consortium in extremely low birth weight infants. PLoS One 6, e27858 (2011).

90. Kalliomäki, M. et al. Distinct patterns of neonatal gut microflora in infants in whom atopy was and was not developing. J. Allergy Clin. Immunol. 107, 129-134 (2001).

91. Hansen, C.H.F. et al. Patterns of early gut colonization shape future immune responses of the host. PLoS One 7, e34043 (2012).

92. Madan, J. et al. Serial analysis of the gut and respiratory microbiome in cystic fibrosis in infancy: interaction between intestinal and respiratory tracts and impact of nutritional exposures. MBio 3, e00251-12 (2012).

93. Martin, R. et al. Early life: gut microbiota and immune development in infancy. Beneficial Microbes 1, 367-382 (2010).

94. Olszak, T. et al. Microbial exposure during early life has persistent effects on natural killer T cell function. Science 336, 489-493 (2012). 
95. Sjögren, Y.M. et al. Influence of early gut microbiota on the maturation of childhood mucosal and systemic immune responses. Clin. Exp. Allergy 39, 1842-1851 (2009).

96. Strachan, D.P. Hay fever, hygiene, and household size. B.M.J. 299, 1259-1260 (1989).

97. Björkstén, B., Naaber, P., Sepp, E. \& Mikelsaar, M. The intestinal microflora in allergic Estonian and Swedish 2-year-old children. Clin. Exp. Allergy 29, 342-346 (1999).

98. Vliagoftis, H., Kouranos, V.D., Betsi, G.I. \& Falagas, M.E. Probiotics for the treatment of allergic rhinitis and asthma: systematic review of randomized controlled trials. Ann. Allergy Asthma Immunol. 101, 570 (2008).

99. Nagalingam, N.A., Cope, E.K. \& Lynch, S.V. Probiotic strategies for treatment of respiratory diseases. Trends Microbiol. 21, 485-492 (2013).

100. Rautava, S., Salminen, S. \& Isolauri, E. Specific probiotics in reducing the risk of acute infections in infancy-a randomised, double-blind, placebo-controlled study. Br. J. Nutr. 101, 1722 (2009).

101. Hojsak, I. et al. Lactobacillus GG in the prevention of nosocomial gastrointestinal and respiratory tract infections. Pediatrics 125 e1171-e1177 (2010).

102. Maldonado, J. et al. Human milk probiotic Lactobacillus fermentum CECT5716 reduces the incidence of gastrointestinal and upper respiratory tract infections in infants. J. Pediatr. Gastroenterol. Nutr. 54, 55-61 (2012)

103. Taipale, T. et al. Bifidobacterium animalis subsp. lactis BB-12 in reducing the risk of infections in infancy. Br. J. Nutr. 105, 409-416 (2011).

104. Sazawal, S. et al. Prebiotic and probiotic fortified milk in prevention of morbidities among children: community-based, randomized, doubleblind, controlled trial. Plos One 5, e12164 (2010).
105. Chen, Y.S., Lin, Y.L., Jan, R.L., Chen, H.H. \& Wang, J.Y. Randomized placebo-controlled trial of lactobacillus on asthmatic children with allergic rhinitis. Pediatr. Pulmonol. 45, 1111-1120 (2010).

106. Abrahamsson, T., Sandberg Abelius, M., Forsberg, A., Björkstén, B. \& Jenmalm, M. ATh1/Th2-associated chemokine imbalance during infancy in children developing eczema, wheeze and sensitization. Clin. Exp. Allergy 41, 1729-1739 (2011).

107. Guillemard, E., Tanguy, J., Flavigny, A.L., De La Motte, S. \& Schrezenmeir, $\mathrm{J}$. Effects of consumption of a fermented dairy product containing the probiotic Lactobacillus casei DN-114 001 on common respiratory and gastrointestinal infections in shift workers in a randomized controlled trial. J. Am. Coll. Nutr. 29, 455-468 (2010).

108. Ezendam, J. \& Van Loveren, H. Lactobacillus casei Shirota administered during lactation increases the duration of autoimmunity in rats and enhances lung inflammation in mice. Br. J. Nutr. 99, 83 (2008).

109. Kawase, M., He, F., Kubota, A., Harata, G. \& Hiramatsu, M. Oral administration of lactobacilli from human intestinal tract protects mice against influenza virus infection. Lett. Appl. Microbiol. 51, 6-10 (2010).

110. Youn, H.-N. et al. Intranasal administration of live Lactobacillus species facilitates protection against influenza virus infection in mice. Antivir. Res. 93, 138-143 (2012).

111. Pellaton, C. et al. Intragastric and intranasal administration of Lactobacillus paracasei NCC2461 modulates allergic airway inflammation in mice. Int. J. Inflamm. 2012, 686739 (2012).

112. Hajishengallis, G., Darveau, R.P. \& Curtis, M.A. The keystone-pathogen hypothesis. Nat. Rev. Microbiol. 10, 717-725 (2012). 